\title{
Measurement of absolute lung volumes by imaging techniques
}

\author{
J. Clausen
}

\begin{abstract}
Measurement of absolute lung volumes by imaging techniques. J. Clausen. CERS Journals Ltd 1997.

ABSTRACT: In this paper, the techniques available for estimating total lung capacities from standard chest radiographs in children and infants as well as adults are reviewed. These techniques include manual measurements using ellipsoid and planimetry techniques as well as computerized systems. Techniques are also available for making radiographic lung volume measurements from portable chest radiographs. There are inadequate data in the literature to support recommending one specific technique over another. Though measurements of lung volumes by radiographic, plethysmographic, gas dilution or washout techniques result in remarkably similar mean results when groups of normal subjects are tested, in patients with disease, the results of these different basic measurement techniques can differ significantly. Computed tomographic and magnetic resonance techniques can also be used to measure absolute lung volumes and offer the theoretical advantages that the results in individual subjects are less affected by variances of thoracic shape than are measurements made using conventional chest radiographs. Eur Respir J 1997; 10: 2427-2431.
\end{abstract}

\author{
Correspondence: J.L. Clausen \\ UCSD Medical Center MC 8378 \\ 200 W Arbor St. \\ San Diego \\ California \\ USA $92103-8378$ \\ Keywords: Computed tomography \\ lung volume measurements \\ thoracic radiography \\ Received: January 301997 \\ Accepted after revision July 151997 \\ This publication evolved from a workshop \\ on measurement of lung volumes sup- \\ ported by the American Thoracic Society \\ and the National Heart, Lung and Blood \\ Institute (Grant No. R13 HL48384), which \\ included participants from the European \\ Respiratory Society.
}

Qualitative assessments of lung volumes are performed unconsciously by most clinicians during review of chest radiographs. More quantitative measurements may enable assessments of lung function to be made from routine radiographs that are often already needed for epidemiological studies or for the care of patients suspected of having lung disease or dysfunction. In subjects with limited ability to co-operate, radiographic lung volumes may be more feasible than physiological measurements; they are also generally less expensive than gas dilution or plethysmographic techniques. Although they carry their own assumptions and limitations, radiographic techniques also obviate some of the uncertainties of physiological measurements of lung volumes, such as the impact of poorly communicating spaces on gas dilution techniques or plethysmographic errors secondary to failure of dynamic pressures measured at the mouth to reflect changes in alveolar pressures.

\section{Conventional chest radiographs}

Although there were a number of earlier efforts to measure lung volumes from chest radiographs, they were less accurate than the method described in 1960 by BARNHARD et al. [1], later modified by LoYD et al. [2]. They considered the thorax as a stack of five ellipsoids for which volumes can be calculated from transverse diameters and heights measured from posteroanterior (PA) and lateral chest radiographs; adjustments are made for magnification factors, and volumes of the heart, intrathoracic tissue and blood, and infradiaphragmatic spaces. Figure 1 illustrates the types of tracings utilized in this technique.
A fundamentally different approach was developed by PratT and KLUGH [4]. It eliminated the need to assume a geometric shape of the thoracic cage representative of all subjects by measuring intrathoracic areas on PA and lateral chest radiographs using planimeters (engineering devices designed to measure the area of irregularly shaped spaces) and empirically relating these areas to measurements of lung volumes on the same subjects, measurements initially obtained from postmortem studies [4] and later [5] from in vivo gas dilution and plethysmographic measurements. Figure 2 illustrates the boundaries used in scribing the thoracic outlines for the planimetry method. The boundaries for the PA view are the same as for the ellipsoid method; for the lateral view, the boundaries include a single diaphragm dome halfway between hemidiaphragms (in contrast with using the highest diaphragm for the ellipsoid technique) and the posterior margin of the vertebral bodies (instead of the posterior interior margins of the ribs).

In a comparison of their planimetric technique with the Barnhard/Loyd ellipsoid method, Harris et al. [5] concluded that their technique was slightly less accurate than the ellipsoid technique but easier and faster to perform. In a study of 48 normal subjects which compared lung volume measurements utilizing the Barnhard/ Loyd ellipsoid and Harris planimetry techniques with measurements done by variable pressure body plethysmography [3], high correlation coefficients with plethysmography ( $\mathrm{r}=0.93$ for ellipsoid and 0.97 for planimetry) and similar means for total lung capacity (TLC) measurements by these three techniques $(6.09,6.06$ and 6.17 $\mathrm{L}$, respectively) were noted. As suggested by BLAND and Altman [6] in their discussion of comparisons of techniques, the analysis of differences between the techniques 


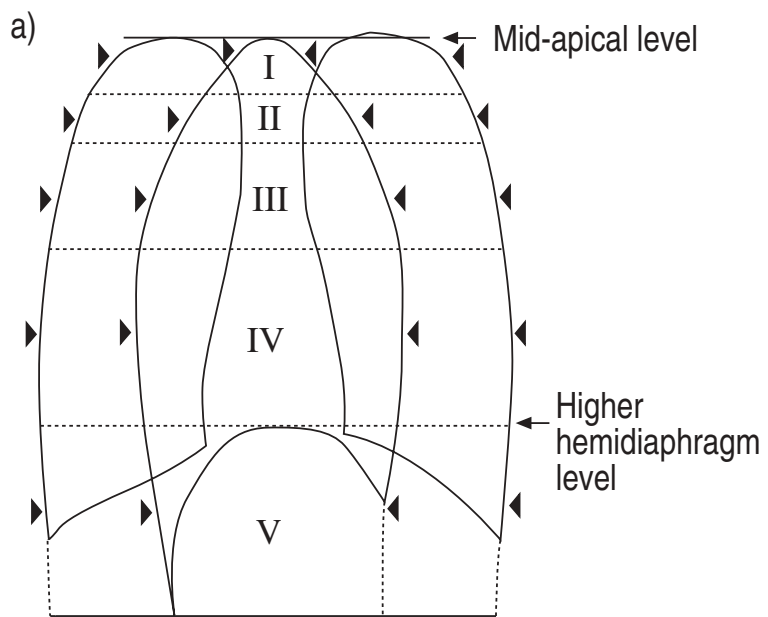

b)
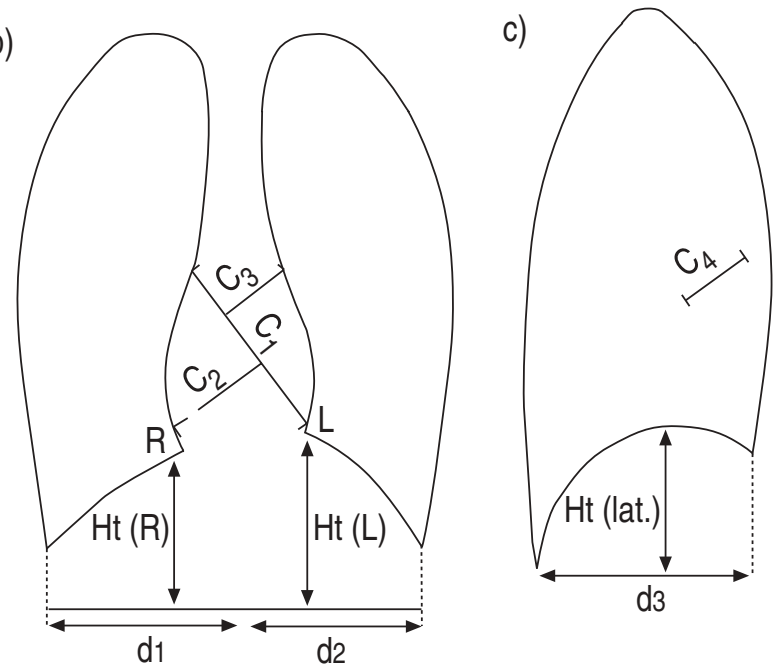

Fig. 1. - a) Posteroanterior and lateral thoracic cage outlines superimposed for delineation of zones $\mathrm{I}-\mathrm{V}$ in the ellipsoid method. b) Diaphragm (d) and heart measurements using the ellipsoid method. c) Lateral diaphragm and heart measurements using the ellipsoid method. Ht: height of hemidiaphragm; $\mathrm{C}_{1}$ : maximal cardiac diameter; $\mathrm{C}_{2}, \mathrm{C}_{3}$ : maximal perpendical distances to the cardiac border, above and below maximal cardiac diamter, respectively; $\mathrm{C}_{4}$ : maximal diameter prependicular to the long axis of the heart. See references [1] and/or [3] for further details of measurement techniques.R: right; L: left (From [3], with permission).

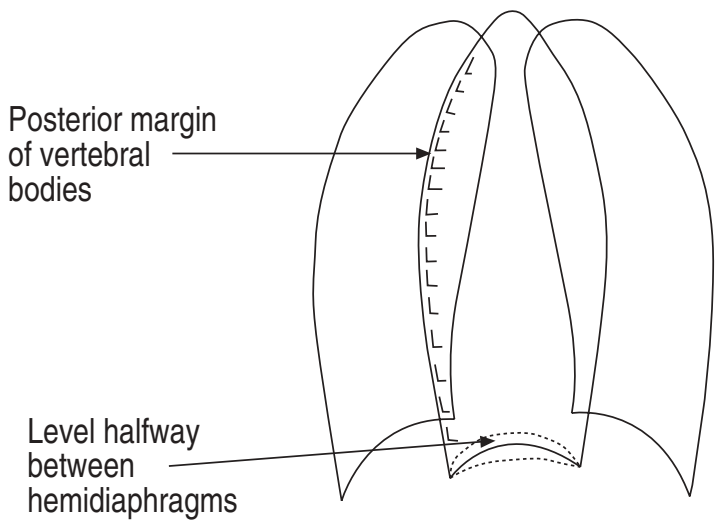

Fig. 2. - Illustration of thoracic cage outlines for the planimetry method with specifics of the boundaries on the lateral view which differ from those used for the ellipsoid methods. (From [3], with permission). in individual subjects indicated more substantial limitations: the standard error of the estimate for the regression comparing radiographic and plethysmographic values was $0.51 \mathrm{~L}$ for ellipsoid and $0.28 \mathrm{~L}$ for planimetric measurements; $25 \%$ of the ellipsoid measurements differed by more than $10 \%$ from plethysmography versus $6 \%$ by planimetry.

Subsequently, a number of papers described automation or computerization of measurements of radiographic lung volumes using both ellipsoid and planimetric techniques. PIERCE et al. [7] described a modification of the ellipsoid method which used a computerized digitizer to measure the volumes of 200 horizontal thoracic slices assumed to be a near-ellipsoid geometric shape described by an equation derived from postmortem crosssectional sections and computed tomography (CT) scans. Adjustments were made for magnification; volumes of the heart, spine, and subdiaphragmatic region; posture; and assumed intrathoracic tissue, blood, and water volumes [7]. Bush and DENISON [8] later proposed improvements for estimating magnification within the Pierce method. In a comparison of techniques, RoDENSTEIN et al. [9] concluded that the Pierce/Bush technique was more accurate than the Barnhard/Loyd technique when compared with plethysmography, but also noted that both radiographic methods had to be interpreted cautiously in individual subjects as differences between the plethysmographic and the Pierce/Bush method were as large as $1.64 \mathrm{~L}$ (20\% of the plethysmographic TLC). Other techniques or equations for measuring radiographic lung volumes have also been described [10].

In 1979, RiEs et al. [11] described a technique for measuring functional residual capacity (FRC) or TLC from supine patients using portable radiography; this paper also described the use of high speed rare earth intensifying screens, very fast X-ray film, and absence of a grid which reduced radiation exposure for these measurements by approximately $90 \%$ as compared with conventional chest radiographs. BLock et al. [12] have described a technique for measuring FRC in supine sleeping adults using portable chest radiographs.

For paediatric applications, the studies are more problematic. Shepard and Seliger [13] concluded that the planimetry technique gave adequate measures of lung volumes on the majority of the 10-12 yr old children they tested, though data from 12 of the 62 children had to be excluded because of unexpectedly wide discrepancies between observed and predicted values. They cautioned that the Barnhard/Loyd elliptical method gave a substantial systematic error when applied to children. Using a special nomogram for predicting lung tissue and blood volumes in smaller children, HILLER et al. [14] found correlation coefficients of 0.93 when ellipsoidradiographic and helium TLCs were compared in adolescents aged 12-19 yrs who either were healthy or had only mild cystic fibrosis. Similar correlation coefficients were observed between plethysmographic and radiographic TLCs [15] both by the ellipsoid method of Barnard/ Loyd and the method described by GAMSU [16] which requires only a PA radiograph and two linear measurements. In this study, it was notable that the $95 \%$ confidence intervals for estimating radiographic TLCs in individual children from the regression lines were greater than $\pm 860 \mathrm{~mL}$, or greater than $\pm 30 \%$ of the median TLC 
observed by plethysmography [15]. SALAM and WARWICK [17] extended the planimetry technique to $4-16$ yr olds and after a comparison of nine different equations with body plethysmographic measurements, identified equations that were optimal at both high and low lung volumes. CAMPBELL [18] compared three different published equations for using planimetry techniques in children aged 5-10 yrs and identified the best as one of two equations developed by SAlEM and Warwick [17]. CUTRERA and HeLms [19] compared radiographic volumes from the Pierce method (as modified by Helms) with helium dilution and plethysmographic volumes and concluded that in children with cystic fibrosis, results from these techniques differed considerably and could not be used interchangeably. More recently, using measurements of lung volumes by body plethysmography and nitrogen washout in 26 infants with bronchopulmonary dysplasia, FUMEY et al. [20] developed regression equations for estimating lung volumes from PA chest radiographs alone or combined with lateral views.

The inter- and intra-observer reproducibility of radiographic lung volume measurements on the same radiographs have been defined by a number studies $[2,7,9]$; representative numbers are intra-observer coefficients of variation $(\mathrm{CV}=$ standard deviation/mean 100$)$ of $0.56 \%$, interobserver $\mathrm{CV}$ of $4.9 \%$ [7]. There are limited data on the reproducibility of TLCs from repeat radiographs; CUTRERA [19] noted four to fivefold larger differences when differences between repeat radiographic and plethysmographic TLCs were compared with the corresponding differences between repeat helium and plethysmographic TLCs in children with cystic fibrosis.

\section{Computed tomography}

In contrast with the inherent limitations of one or twoview radiographic techniques, CT and magnetic resonance imaging (MRI) techniques offer the potential for accommodating for individual variances in chest wall shape. As with radiographic techniques, CT and MRI also offer the potential for volume measurements of specific regions or sections of lung [21]. In addition to thoracic cage volumes, CTs can provide estimates of lung tissue and air volumes and can also estimate the volumes of lung occupied by increased density (as in patchy infiltrates) or decreased density (as in emphysema or bullae) [22-24]. CT methods utilize thresholds of attenuation numbers for lung and adjacent tissues of ribs, diaphragms, and the heart to define the boundary of the thoracic cage; in some systems this boundary can be modified by the reviewer. Lung volumes are then computed from the sums of the lung areas multiplied by the thickness of sections and the number of sections [21, $22,24]$. In the literature, the details of how the CT volumes are measured are often sketchy, in part because much of the CT computational methodology utilizes device-specific software considered proprietary. CANN [25] has pointed out that not all CT scanners are equally suited for quantitative applications, and results on one machine may not be comparable to those of another unless correction factors are used.

In one of the few studies comparing volumes computed from both chest radiographs and CTs, in the 21 children studied, comparable correlations were observed for CT and radiographic measurements as compared with plethysmographic TLCs [15].

The radiation doses involved with conventional CT preclude widespread use of this technology for measuring lung volumes unless the CTs are also obtained for other purposes. If, however, fine detail of parenchymal images is not needed, the CT procedure could be modified to substantially reduce radiation dose. Advances in ultrafast or spiral CTs are resulting in significant decreases in radiation doses and offer capabilities for dynamic assessments of regional lung volumes in units as small as individual lobules.

\section{Magnetic resonance imaging (MRI)}

Chapman et al. [26] demonstrated the potential for measuring thoracic volumes using MRI in unsedated infants; use of the ultrafast echo planar MRI permitted collection of 400 thoracic images within 5 min (far more images than are required for thoracic volume measurements). Improvements in MRI technology will permit generation of images from single breaths. As with CT [21-23, 27], MRI also offers the potential for measuring lung volumes of specific lungs or regions of the lungs as well as adjusting for estimates of lung water and tissue. However, in spite of the advantage of absence of radiation exposure, use of MRI for measuring thoracic volumes will be limited by its considerable cost.

\section{Controversies and critical questions}

1. Can we make specific recommendations for standardizing imaging techniques for measurements of lung or thoracic volumes?

For children and infants, because of the limited number of radiographic and physiological studies of lung volumes in healthy infants, the real or uncertain limitations in "gold-standard" physiological measurements of lung volumes in infants with lung disease, and the paucity of studies which have compared with sufficient power the radiographic methods available in children, no specific techniques can be recommended as "goldstandards" at this time. The most promising methods would seem to be the technique developed by FUMEY et al. [20] for infants and the planimetry equation for children developed by SALAM and WARWICK [17] which was identified as optimal from the comparison by CAMPBELL [18]. In infants, variations in thymus size introduce significant problems for both planimetry and ellipsoid techniques. Also problematical are estimates for lung tissue volume in infants and children.

For adults, because of a lack of studies which have compared all three basic radiographic techniques (Harris, Barnhard, and Pierce), no single technique can be recommended over another. Between the two basic "ellipsoid-like" techniques, the comparison by RoDENSTEIN et al. [9] indicates that the Pierce technique is superior; the Pierce method also offers the advantages of more rapid computerized measurements. Whichever method is used, careful attention must be paid to matching the techniques originally described, especially in regards to 
magnification factors and specific anatomic guidelines for defining thoracic outlines.

Because of the paucity of specific details regarding CT techniques and the lack of adequate comparison studies, no single technique can be recommended. For MRI there is too little experience to make specific recommendations regarding optimal techniques. For both CT and MRI, advances in the speed of imaging and thoughtful selection of the number of images required will significantly affect the potential for use of these techniques for lung volume measurements in the future.

2. Are the inspirations to TLC achieved during routine chest radiographs sufficiently close to those achieved in pulmonary function laboratories where patients are specially instructed to make maximal inspirations?

CRAPO et al. [28] observed that in 19 healthy subjects, radiographic TLCs taken after routine coaching instructions for chest radiographs averaged $95.5 \%$ of the radiographic TLCs measured after special coaching. In four out of 19 subjects, however, the special coaching TLCs were more than $0.60 \mathrm{~L}$ larger than TLCs after routine radiograph instructions.

In an epidemiological study, KILBURN et al. [29] noted that $13 \%$ of subjects had radiographic evidence of inadequate inspirations (diaphragm above the ninth posterior midintercostal space) on standard chest radiographs and $90 \%$ of such subjects achieved better inspiration after encouragement to maximize inspirations.

It is likely that the adequacy of inspiratory efforts during "routine" radiographs is highly site and subject specific; when optimal accuracy is required, both the subject and the radiology technician should be instructed regarding inspiratory techniques.

3. Are single radiographic lung volume measurements in individual patients sufficiently accurate to be clinically useful?

Although the mean TLCs from groups of normal subjects may be very close when radiographic and plethysmographic techniques are compared $[2,3,5,9]$ and the correlation coefficients quite high, differences between plethysmographic and radiographic TLCs of more than $20 \%$ in normal subjects have been reported $[1,3,5,7$, $9,30]$, as have standard deviations of $400-800 \mathrm{~mL}$ around regression lines comparing radiographic with plethysmographic or gas dilution TLCs $[5,11,15]$, indicating that individual radiographic lung volumes may be sufficiently inaccurate to limit their clinical usefulness. This conclusion does, however, assume that the plethysmographic or gas dilution measurements are always more accurate and/or reproducible in normal subjects than radiographic TLCs, an assumption with some support from the data of Cutrera and Helms [19]. This uncertainty about the relative reliability of radiographic TLC measurements might be resolved if studies of repeated measurements of plethysmographic and gas dilution and radiographic volumes were performed in those with the largest discrepancies and compared with CT measurements of volumes.

4. How accurate are imaging techniques for measuring lung volumes in patients with infiltrates or other airspace-occupying lung disease?
Although plethysmographic, gas dilution, and radiographic techniques can give lung volumes that are remarkably similar in normal subjects, they measure fundamentally different spaces which can differ substantially in patients with lung disease. The planimetric technique relies on regression equations developed from physiological measurements of lung volumes in normal subjects. In patients with lung disease with significant amounts of airspace-occupying tissue (e.g. pneumonia with consolidation; severe interstitial fibrosis), some [31, $32]$ but not all $[33,34]$ studies have observed that radiographic lung volumes may be substantially greater than the compressible gas volumes or communicating gas volumes measured by plethysmography or gas dilution techniques, respectively; the differences between these studies may reflect the severity of lung disease in the study patients. With the ellipsoid techniques, the values reported are generally adjusted for assumed values of intrathoracic blood and fluids and lung tissue values appropriate for normal subjects. CT and MRI techniques offer the potential for measuring intrathoracic volumes and estimating lung gas volumes after subtraction of estimates of fluid and tissue volumes derived from measurements of image density.

\section{References}

1. Barnhard HJ, Pierce JA, Joyce JW, Bates JH. Roentgenographic determination of total lung capacity. Am J Med 1960; 28: 51-60.

2. Loyd HM, String T, DuBois AB. Radiographic and plethysmographic determination of total lung capacity. Radiology 1966; 86: 7-14.

3. Clausen JL, Powell Zarins L. Estimation of lung volumes from chest radiographs. In: Clausen JL, ed. Pulmonary Function Testing. Guidelines and Controversies, Equipment, Methods, and Normal Values. New York, Academic Press Inc., 1982; pp. 155-163.

4. Pratt PC, Klugh GA. A method for the determination of total lung capacity from posteroanterior and lateral chest roentgenograms. Am Rev Respir Dis 1967; 96: 548-552.

5. Harris TR, Pratt PC, Kilburn KH. Total lung capacity measured by roentgenograms. Am J Med 1971; 50: 756-763.

6. Bland JM, Altman DG. Statistical methods for assessing agreement between two methods of clinical measurement. Lancet 1986; 1: 307-310.

7. Pierce RJ, Brown DJ, Holmes M, Cumming G, Denison DM. Estimation of lung volumes from chest radiographs using shape information. Thorax 1979; 34: 726-734.

8. Bush A, Denison DM. Use of different magnification factors to calculate radiological lung volumes. Thorax 1986; 41: 158-159.

9. Rodenstein DO, Sopwith T, Denison DM, Stanescu DC. Reevaluation of the radiographic method for measurement of total lung capacity. Bull Eur Physiopathol Respir 1985; 21: 521-525.

10. Teklu B, Gray WM, Mills RJ, Moran F. A critical appraisal of a rapid radiographic method of determining total lung capacity. Scott Med J 1986; 31: 99-102.

11. Ries AL, Clausen JL, Friedman PJ. Measurement of lung volumes from supine portable chest radiographs. $J$ Appl Physiol 1979; 47: 1332-1335.

12. Block AJ, Bush CM, White C, Boysen PG, Wynne JW, Taasan VC. A radiographic method for measuring steadystate functional residual capacity in the supine patient. 
A method suitable for sleep studies. Am Rev Respir Dis 1981; 124: 330-332.

13. Shephard RJ, Seliger V. On the estimation of total lung capacity from chest $\mathrm{x}$-rays; radiographic and helium dilution estimates on children aged 10-12 years. Respiration 1969; 26: 327-336.

14. Hiller EJ, Kirkpatrick JA, Huang N. Radiographic determination of TLC in patients with cystic fibrosis. J Pediatr 1971; 78: 435-440.

15. Schlesinger AE, White DK, Mallory GB, Hildeboldt, Huddleston CB. Estimation of total lung capacity from chest radiography and chest $\mathrm{CT}$ in children: comparison with body plethysmography. Am J Roentgenol 1995 ; 165: 151-154.

16. Gamsu G, Shames DM, McMahon J, Greenspan RH. Radiographically determined lung volumes at full inspiration and during dynamic forced expiration in normal subjects. Invest Radiol 1975; 10: 100-108.

17. Salam H, Warwick WJ. Measurement of total lung capacity by a roentgenography-planimetry method in children 4-16 years of age. Respiration 1978; 36: 177182.

18. Campbell SC. Estimation of total lung capacity by planimetry of chest radiographs in children 5 to 10 years of age. Am Rev Respir Dis 1983; 127: 106-107.

19. Cutrera R, Helms P. Retrospective estimation of values for total lung capacity by plethysmography, helium gas dilution, and chest radiography in patients with cystic fibrosis. Thorax 1988; 43: 931-932.

20. Fumey MH, Nickerson BG, Birch M, McCrea R, Kao LC. A radiographic method for estimating lung volumes in sick infants. Pediat Pulmonol 1992; 13: 42-47.

21. Sallustio G, Bray A, Colavito MH, Pirronti T, Marano P. High resolution computed tomography (HCRT) and new perspectives in functional radiology of the lung. Rays 1997; 22: 157-182.

22. Coxson HO, Hogg JC, Mayo JR, et al. Quantification of idiopathic pulmonary fibrosis using computed tomography and histology. Am J Respir Crit Care Med 1997; 155: $1649-1656$.
23. Johnson RL Jr, Cassidy SS, Grover R, et al. Effect of pneumonectomy on the remaining lung in dogs. $J$ Appl Physiol 1991; 70: 849-858.

24. Archer DC, Coblentz CL, deKemp RA, Nahnmias C, Norman G. Automated in vivo quantification of emphysema. Radiology 1993; 188: 835-838.

25. Cann CE. Quantitative CT applications: comparison of current scanners. Radiology 1987; 162: 257-261.

26. Chapman B, O'Callaghan C, Coxon R, et al. Estimation of lung volume in infants by echo planar imaging and total body plethysmography. Arch Dis Child 1990; 65: 168-170.

27. Friedman PJ, Peters RM, Botkin MC, Brimm JE, Meltvedt RC. Estimation of the volume of lung below the left atrium using computed tomography. Crit Care Med 1986; 14: $182-187$.

28. Crapo RO, Montague T, Armstrong JD. Inspiratory lung volume achieved on routine chest films. Invest Radiol 1979; 14: 137-140.

29. Kilburn KH, Warshaw RH, Thornton JC, Thornton K, Miller A. Predictive equations for total lung capacity and residual volume calculated from radiographs in a random sample of the Michigan population. Thorax 1992; 47: 519-523.

30. Barrett WA, Clayton PD, Lambson GR. Computerized roentgenographic determination of total lung capacity. Am Rev Respir Dis 1976; 113: 239-244.

31. Teklu B, Gray WM, Mills RJ, Moran F. A critical appraisal of a rapid radiographic method of determining total lung capacity. Scott Med J 1986; 31: 99-102.

32. Clausen JL, Zarins LP, Ries AL. Measurements of abnormal increases in pulmonary tissue in restrictive lung disease. Am Rev Respir Dis 1978; 117 (Suppl.): 322.

33. Ferris BG. Use of chest radiography in epidemiologic studies of nonoccupational lung diseases. Am Rev Respir Dis 1978; 118 (number 6, part 2): 89-112.

34. Kilburn KH, Warshaw RH. Total lung capacity in asbestosis: a comparison of radiographic and body plethysmographic methods. Am J Medical Sciences 1993; 305: 84-87. 